\title{
LA CALIDAD DEL AGUA EN LA CUENCA DEL RIO PILOÑA (ASTURIAS)
}

\author{
F. González Alvarez-Buylla, A. Miramda Braga, J.R. Alonso Fernández, M.A. Abella García \\ Confederación Hidrográfica del Norte de España. Oviedo.
}

Palabras clave: Physical and chemical parametres, macroinvertebrates, flora, quality index.

\author{
ABSTRACT \\ THE QUALITY OF THE WATER IN THE PILOÑA BASIN (ASTURIAS)
}

Out from the data of a study of physical and chemical parametres, a count of coliforms and the use of benthic macroinvertebrates and algae as bioindicators, the most polluted areas have been determined and some resulting biological effects have been observed.

\section{INTRODUCCION}

La cuenca del río Piloña tiene aproximadamente $508,9 \mathrm{Km}$ ' de superficie; está formada principalmente por los ríos Viao y Fuensanta, que forman el río Piloña, que a su vez tiene como afluentes principales a los ríos De la Marea, Espinaredo, Borines, Color, Cúa y Mampodre. El río Piloña con aproximadamente $28 \mathrm{Km}$. de recorrido, es el principal afluente del río Sella (figura 1).

El sustrato geológico es bastante variado en su mayoría con lecho calizo salvo algunas bandas de areniscas y pizarras. El clima de la zona corresponde al tipo atlántico-europeo. salvo algunas pequeñas áreas donde nacen algunos de los afluentes del río $\mathrm{Pi}$ loña (con altitudes superiores a los 800 m.s.n.m.), donde el clima dominante corresponde al tipo centro-europeo.

En el presente estudio con objeto de ofrecer una perspectiva amplia de toda la cuenca del río Piloña, consideramos como zona de cabecera el nacimiento del rio Viao (440 m.s.n.m.); de esa forma, a la vista del perfil del río (figura 2) se puede constatar que éste no es el más típico de un río de la zona norte dado el suave desnivel desde el nacimiento hasta la desembocadura.

Sin lugar a dudas, la mayor carga contaminante que recibe la cuenca estudiada (figura 1), procede de los vertidos urbanos de las principales localidades (Nava, Intiesto, Villamayor, Sevares, Arriondas). Aparte de estos, constatar la existencia de tres piscifactorías y diversas fábricas de muebles y productos

Limnética 2: 225-234(1986)

O Asociación Española de Limnología, Madrid. Spain alimenticios. El río Piloña y sus afluentes a lo largo de su recorrido, reciben una polución equivalente de:

Numero de habitantes en 1984: 11.010

$\mathrm{DBO}_{5} \mathrm{Kg} . /$ día: 748

M.E.S. (Materia en susp.) Kg./dia: 990

El presente trabajo consiste fundamentalmente en un estudio de la calidad del agua de la cuenca del río Piloña, y establecer complementariamente a estos datos algunos efectos biológicos particularmente en las zonas donde la contaminación es más elevada.

\section{MATERIAL Y METODOS}

Nos interesaba reflejar el estado de la calidad del agua en un número elevado de estaciones de muestreo; para la elección de éstas hemos tenido en cuenta la localización de vertidos potencialmente contaminantes, la desembocadura de los diversos afluentes y los resultados de un estudio biológico previo que confirmaron las apreciaciones iniciales de cara al establecimiento cie 48 estaciones de muestreo (figura 1).

Durante el año 1984 hemos llevado a cabo tres campañas de muestreo: mientras que en invierno y verano hemos muestreado en la totalidad de las estaciones, en primavera no lo hemos hecho en los afluentes del río Piloña. En el campo hemos utilizado para medir la velocidad del agua el molinete universal OOT C31 (10.002) A.OOT. Kemtew, y liemos tomado muestras de agua para realizar análisis químicos y recuentos bacteriológicos. Al mismo tiempo hemos tomado muestras de los organismos vivos (fauna 


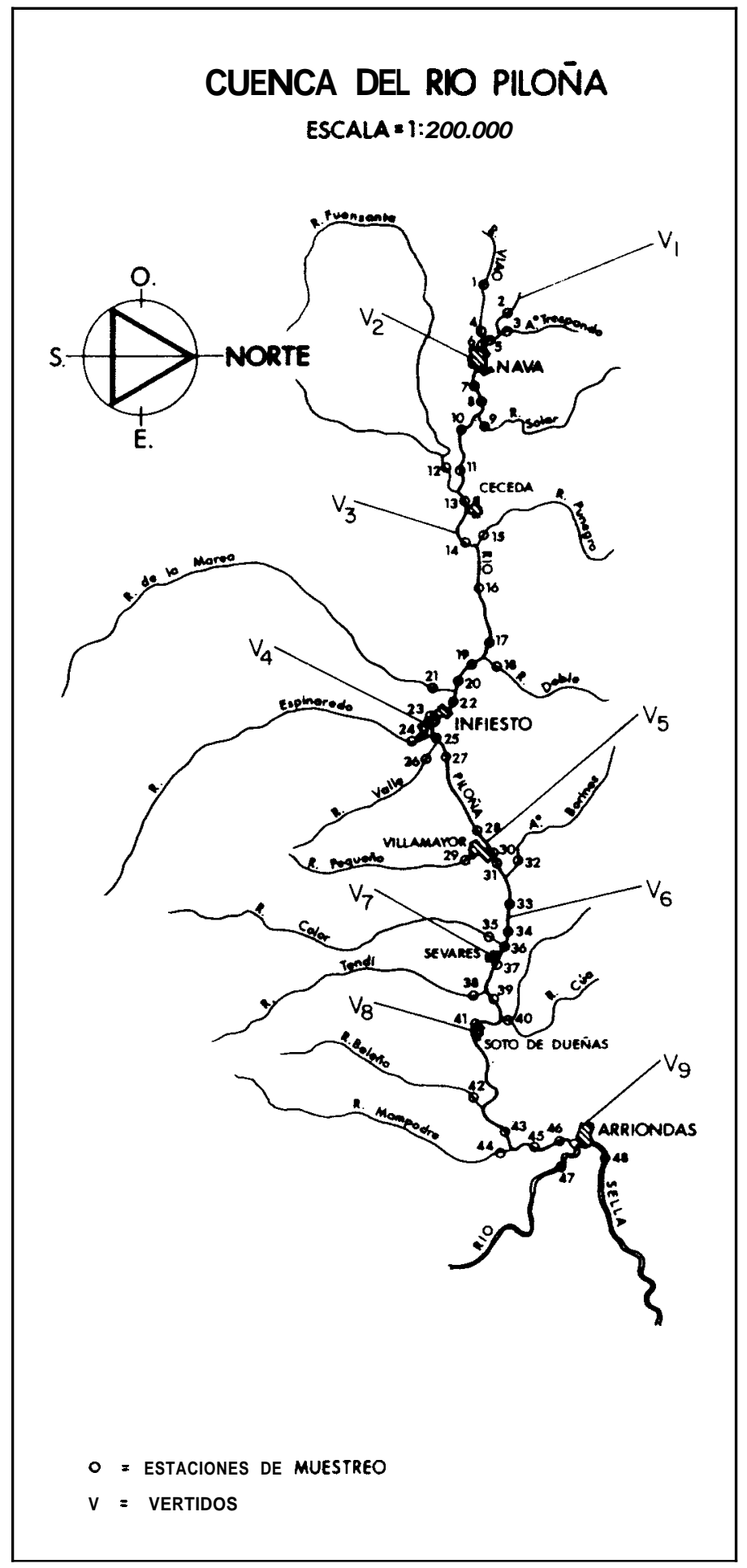

Figura 1.-Mapa de la cuenca del río Piloña con la localización de estaciones de muestreo y vertidos. A map of the basin of the river Piloña with the location marked of the testing stations and waste outlets 
PERFIL LONGUITUDINAL - RIO PILOÑA

ESCALA H. 1:200.000

V. 1: 10.000

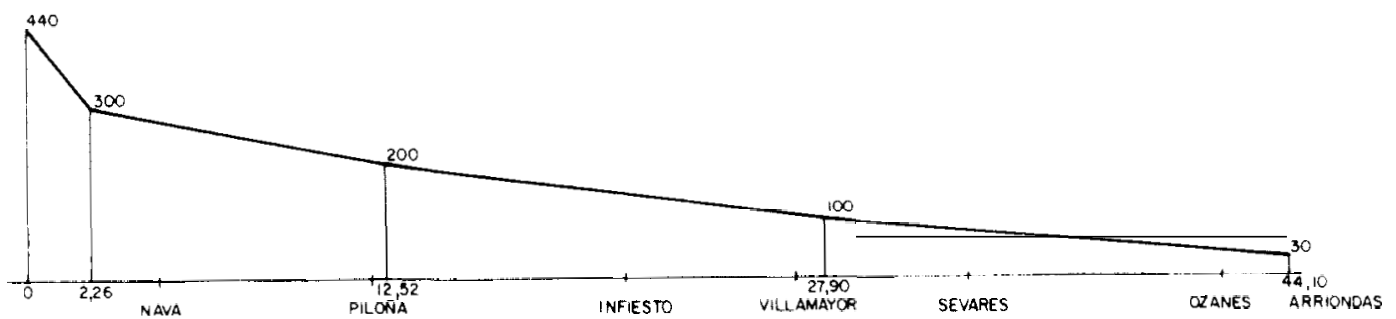

Figura 2.-Perfil longitudinal del río Viao-Piloña.

Section ofthe river Piloña.

RIO PILOÑA

ESTACION DE AFOROS - AN

DE VILLAMAYOR N2 303

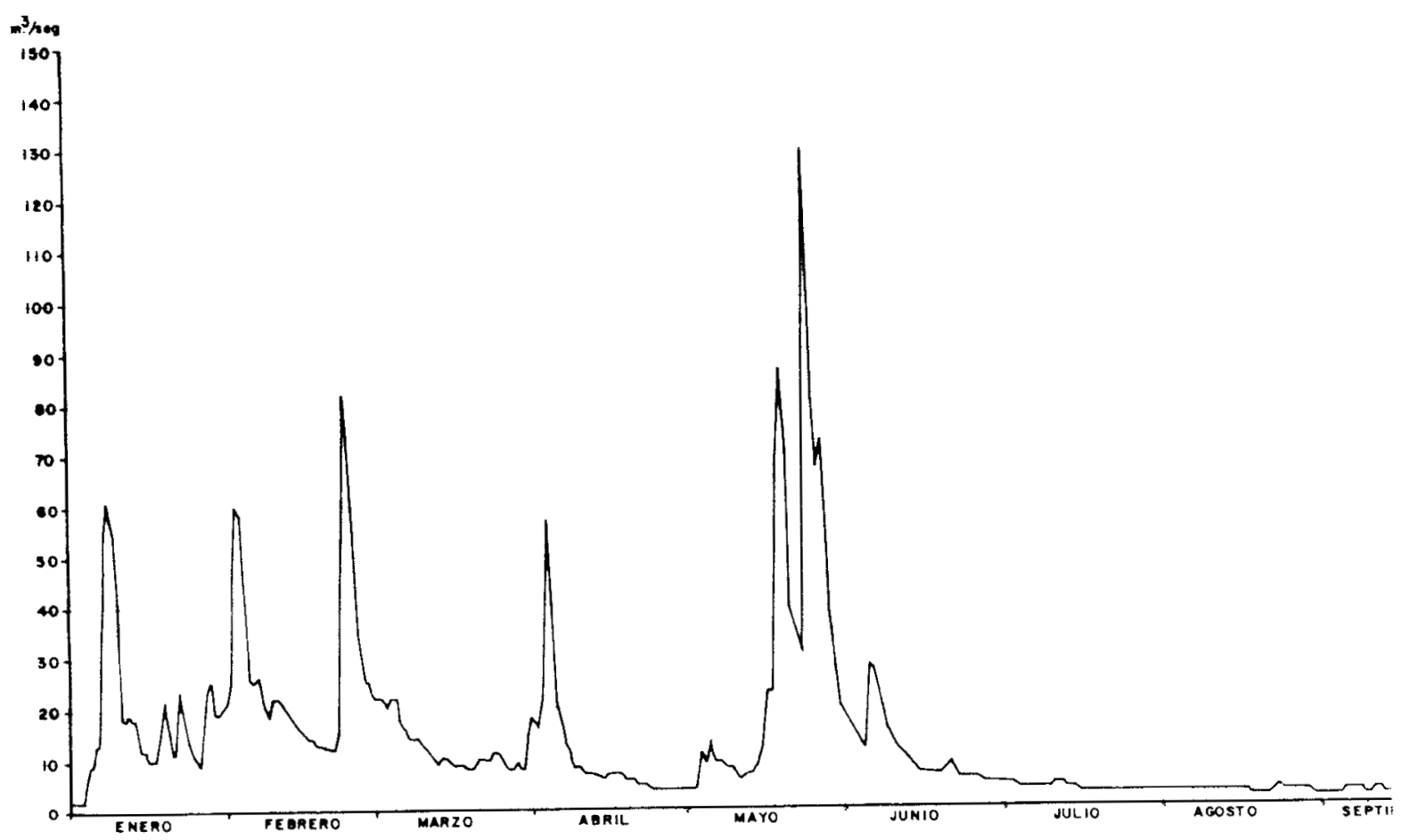

Figura 3.-Evolucióri del caudal en la estación de aforos de Villamayor (E-33), enero-spetiembre de 1984 Changes in the volume at the gauging station Villamayor (E-33)january-september of 1984. 


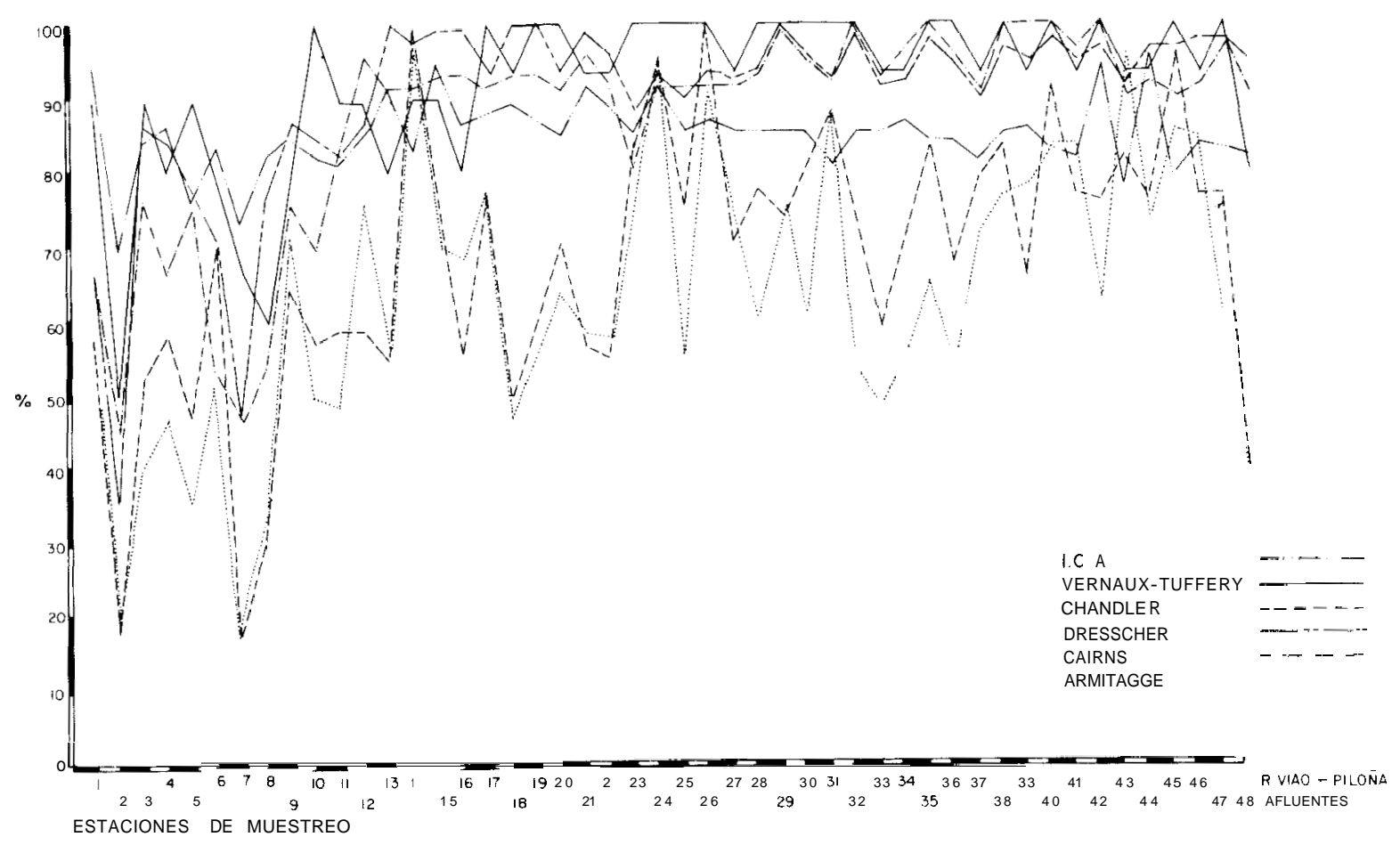

CUENCA RIO VIAO-PILOÑA INDICES DE CALIDAD DEL AGUA - INVIERNO DE 1984

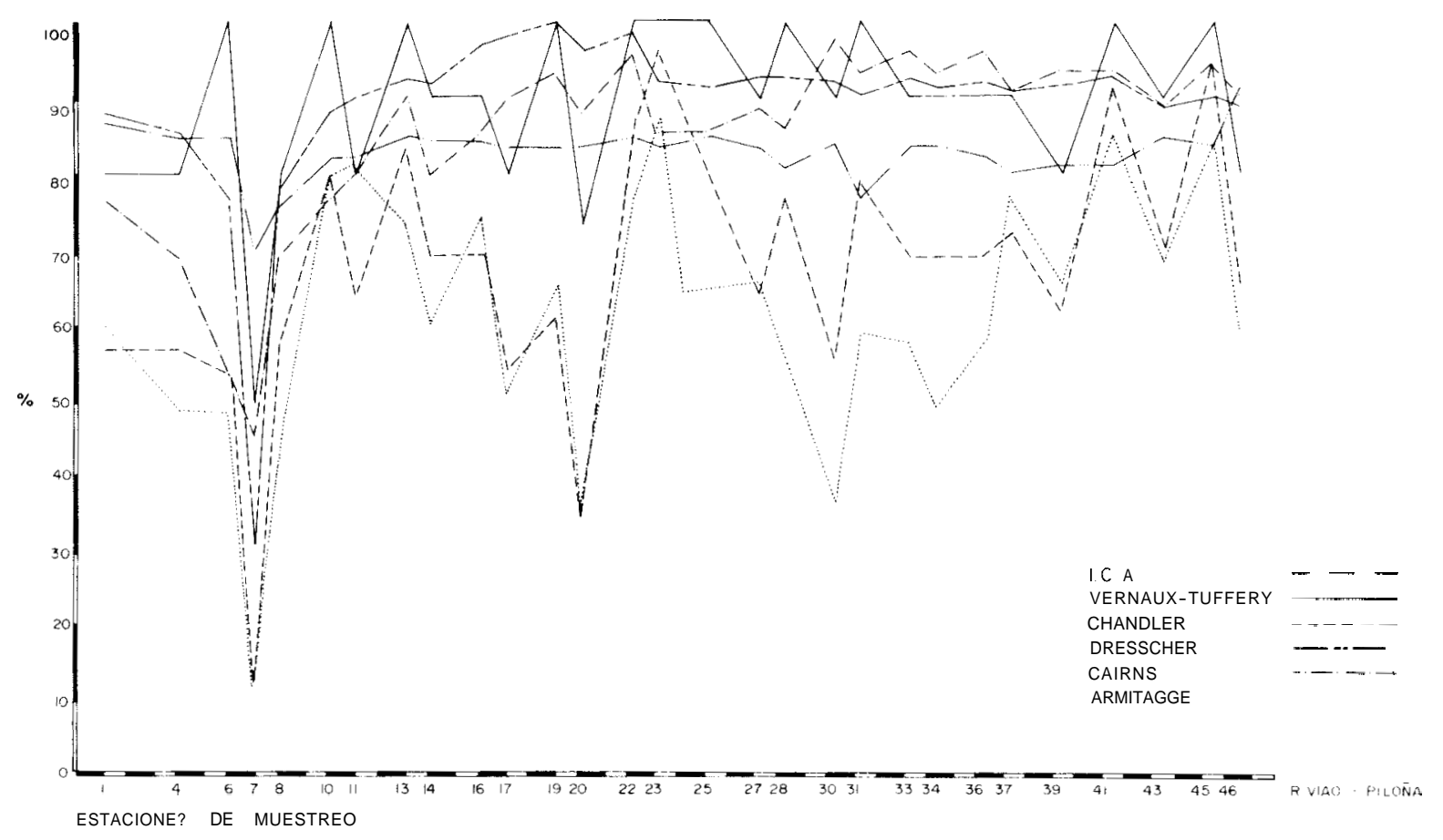

CUENCA RIO VIAO-PILON̄A INDICES DE CALIDAD DEL AGUA - PRIMAVERA DE 1984 


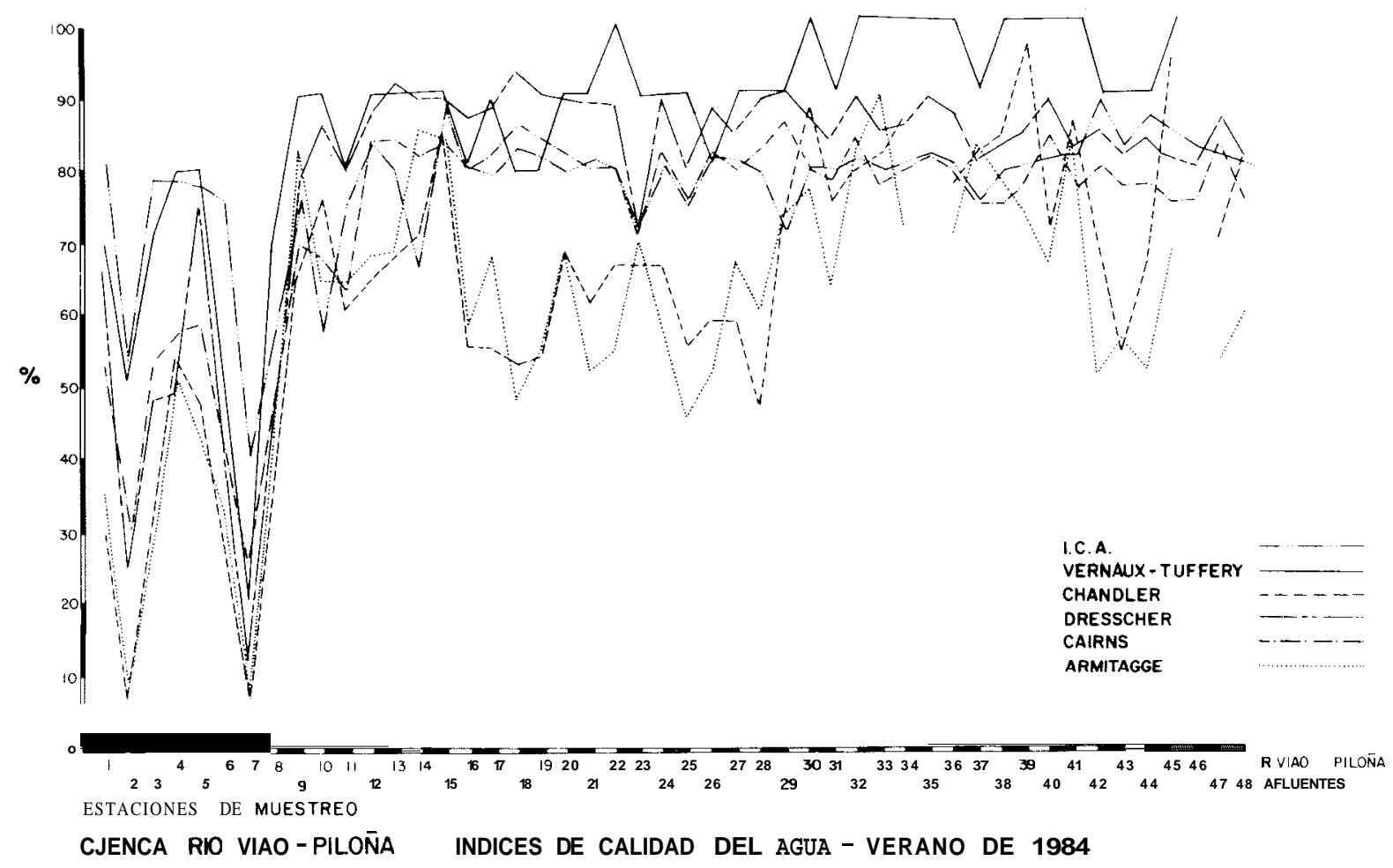

Figura 4.-Indiccs de calidad en invierno, primavera y verano. (1984) Quality indcx in winter spring and summer. (1984).

y flora): la captura de macroinvertebrados bfnticos ha sido realizada con una red Surber, de 300 micras de luz de malla, y para la recogida de micrófitos nos hemos ayudado de una manga de 180 micras de luz de malla y de una espátula para raspar piedras del fondo, objetos y vegetales sumergidos. En un cuaderno de campo hemos tomado datos concernientes a la vegetación acuática y de los márgenes.

Junto al caudal y la temperatura hemos realizado las siguientes determinaciones físico-quimicas: Oxígeno disuelto, materia en suspensión, $\mathrm{pH}$, conductividad, DRO, , DQO. detergentes. amonio, ortofosfatos, nitratos, nitritos, cloruros, sulfatos. dureza, calcio. T AC (alcalinidad total) y TOC (carbono orgánico total). Para realizar estas determinaciones, así como la del número de coliformes totales, se han seguido las normas del Standar Methods (1980) y Degremont (1979).

A partir de la concentración ponderada de los parámetros físico-químicos y del número de coliformes totales. hemos obtenido para cada estación de muestreo el índice de calidad de las aguas (I.C.A.) Mingo, J. (1980). 'También en cada estación de muestreo, a partir de los macroinvertebrados recolectados ha sido aplicado el I.B. (Verneaux, J. \& Tuffery, (i.. 1967). basado en el valor indicador de algunos taxa y la riqueza de unidades sistemáticas de la comunidad; el B.S. (Chandler, J.R. 1970), que define cinco niveles de abundancia para los organismos capturados en un tiempo standard de cinco minutos. Asimismo utilizando también los macroinvertebrados como bioindicadores ha sido aplicado el B.M.W.P. (Armitage, P.D. et al., 1983), que estima solaniente la presencia de deterniinadas familias.

Igualmente en cada una de las estaciones muestreadas hemos obtenido el cociente sapróbico (Dresscher. G.N. \& Mark. H. 1976) donde se considera una serie de grupos como indicadores (ciliados, euglenoficcas. diatomeas). También hemos tenido en cuenta la diversidad de las diatomeas y hemos aplicado el S.C.I. (Cairns et al. 1968).

\section{RESULTADOS}

En la tabla 1 aparecen los resultados de las determinaciones físico-químicas y el número de coliformes totales durante el invierno. primavera y verano a partir de las cuales han sido elaborados los ICA correspondientes que aparecen representados en la figu- 

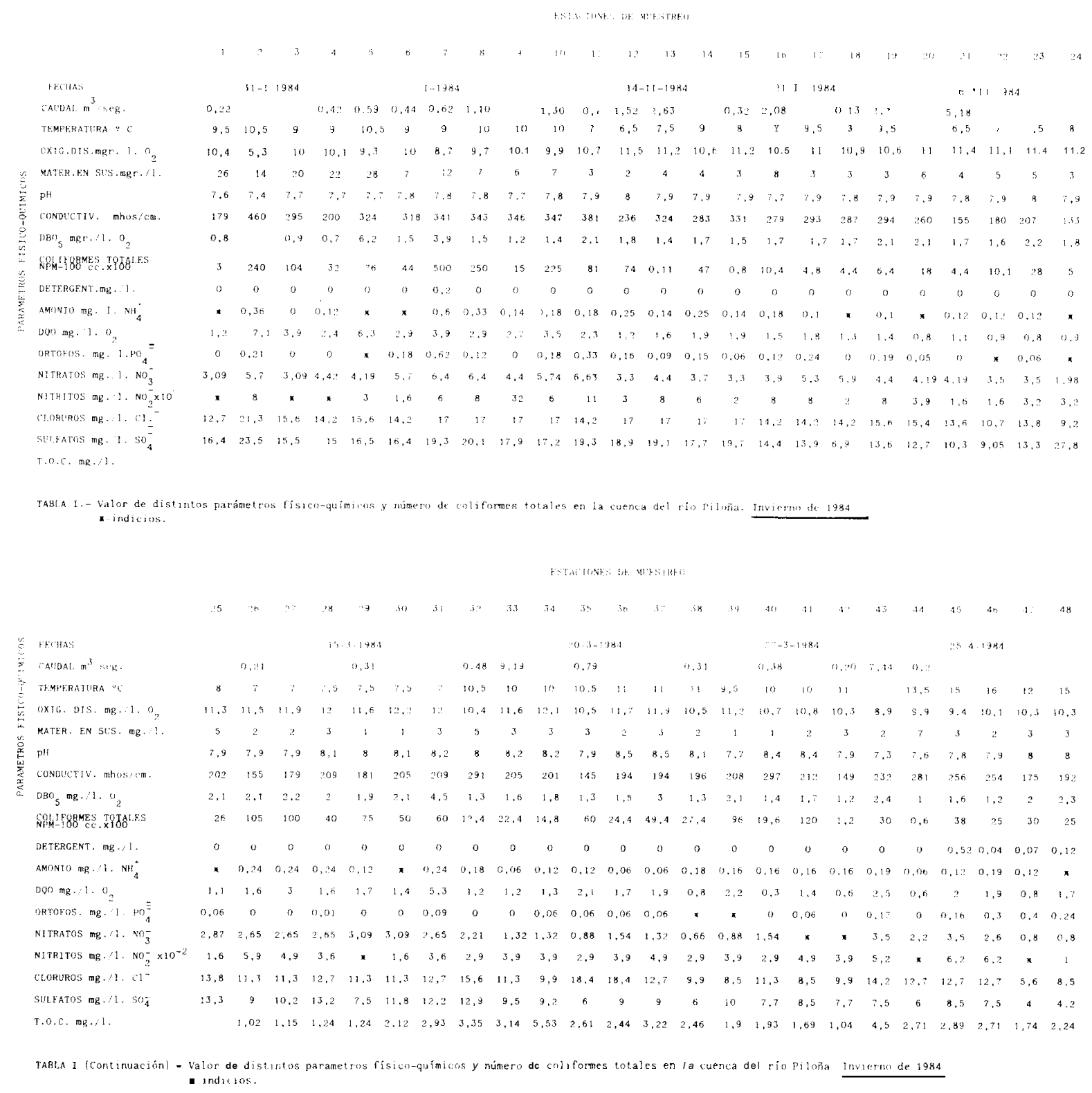

ra 4, junto con los resultados obtenidos al aplicar los índices biológicos en donde se expresa el valor porcentual de cada índice respecto al valor máximo de su rango de variación. En aquellos índices cuyo valor máximo no está explícito B.S., B.M.W.P., S.C.I., se ha elegido como tal el más alto encontrado.

En la tabla 2 aparece reflejada la distribución de los grupos faunísticos y florísticos en las distintas estaciones de muestreo según los diferentes periodos del año estudiados. En el caso de grandes grupos faunisticos como plecóptera, efemeróptera, etc., se da el número de especies de cada uno de los órdenes presentes en cada estación de muestreo. En el caso de ciliados y de grandes grupos vegetales (cianofíceas, euglenofíceas, diatomeas, etc.), se hace referencia a la mayor o menor abundancia de cada uno de ellos en cada una de las estaciones de muestreo, utilizando para ello una escala subjetiva de frecuencias $(+=$ individuos aislados; 1 = poco abundante; $\mathbf{3}=$ abundante; 5 = muy abundante).

Los catálogos faunístico y floristico se encuentran ampliamente detallados en los trabajos que hacen referencia a la utilización de macroinvertebrados y algas dulceacuícolas como indicadores biológicos de al 


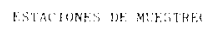

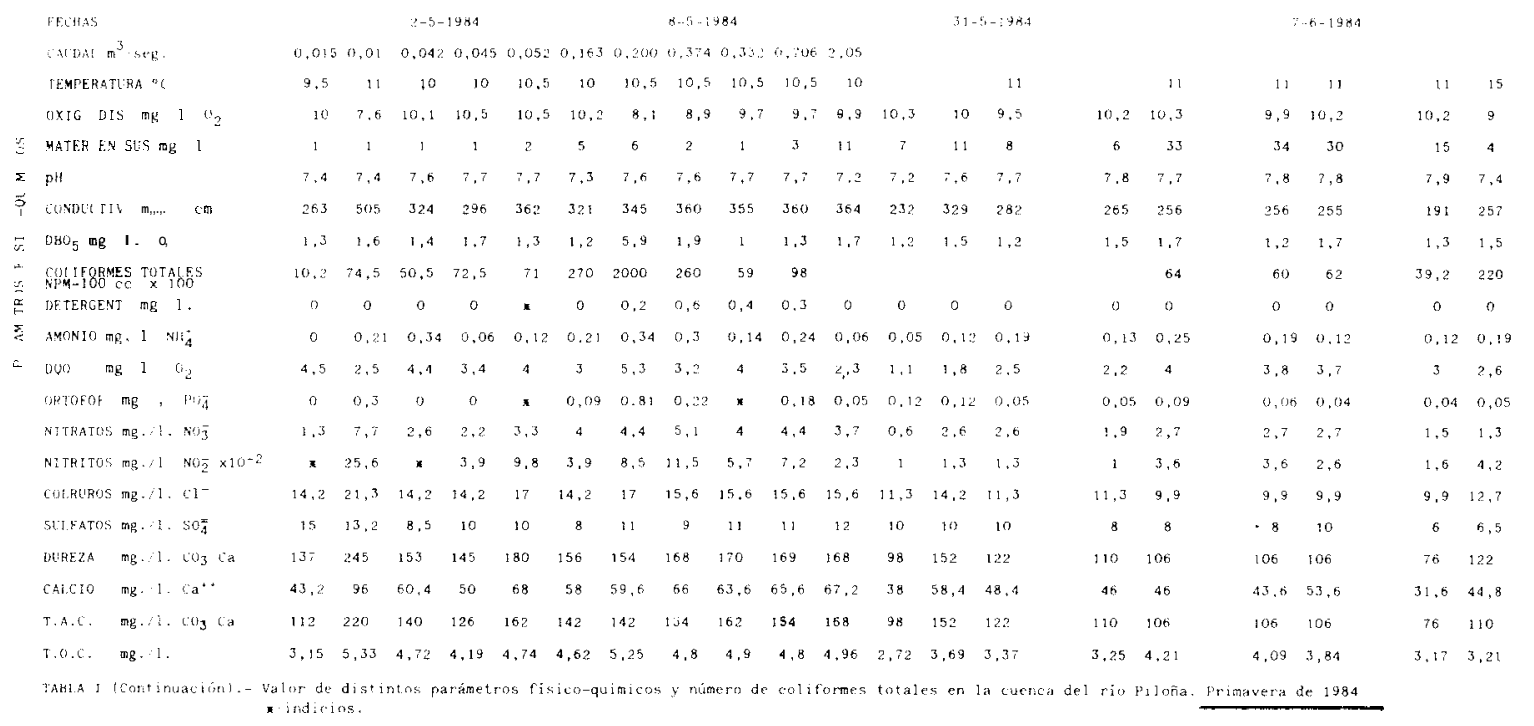

\begin{tabular}{|c|c|c|c|c|c|c|c|c|c|c|c|c|c|c|c|c|}
\hline & & & $\therefore-1+24$ & & & & & $3.4: 9$ & & & & & & & $11-1984$ & \\
\hline$\because \cdots n$ & & & & & & $1, \ldots$ & & & & & & $x, 38$ & & & & \\
\hline$: 4: 16: 1: 1$ & $i$ & 15,8 & in & $1 \ldots \ldots$ & $\therefore$ & $\cdots$ & 1. & $: n$ & $m, n$ & 16,5 & 16,5 & in & & ik & 1.5 & \\
\hline 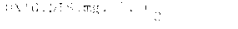 & J.' & 4,1 & $x, 7$ & 4.4 & 4. 1 & $\mathrm{a}$ & ${ }^{9}$ & a & $x, 6$ & 8.5 & 8,8 & 9.3 & ift & $4: 1$ & 16.3 & 10.3 \\
\hline 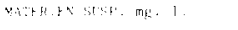 & 1 & : & 3 & 3 & s. & 4 & 4 & 4 & 4 & 5 & ${ }^{4}$ & 4 & 1 & $=$ & i & 1 \\
\hline 鬲 & $T, t$ & 3.7 & $\therefore$ & $\therefore, 9$ & $\therefore+i$ & $\because 8$ & $r i$ & $\because, 8$ & $\therefore 7$ & 7,8 & 7,8 & 3.5 & 7.9 & 8.1 & 8 & 8.1 \\
\hline (स) & 241 & 234 & 240 & 231 & 236 & 258 & 260 & 25.4 & 250 & 255 & $2 t 00$ & 259 & 262 & 255 & 184 & 207 \\
\hline 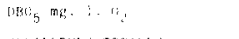 & 1.1 & 1.5 & 2.1 & 1,1 & 4,5 & $1, \mathrm{i}$ & 1,1 & 1,5 & $2, ?$ & 1,1 & 1,5 & 1,7 & 1.4 & 1.6 & 1.6 & 1.9 \\
\hline 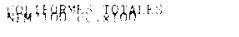 & 180 & $200)$ & & 110 & 130 & 24 & 100 & 98 & $12 t$ & 126 & 134 & so & 86 & 1,1 & 64 & 140 \\
\hline DE:JEHGEN_ $\mathrm{gg}, 1$. & 0 & 0 & 0 & 0 & 0 & 0 & o & 0 & 0 & 0 & 0 & $x$ & 0 & 0 & 0 & 0 \\
\hline 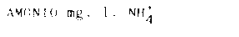 & 0.14 & $0.1:=$ & 0.3 & 0,06 & $0.0 \%$ & 0 & 0 & 0 & 0 & a & 0 & 0.05 & 0.05 & 0,04 & 0.04 & 0.05 \\
\hline 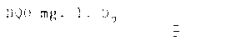 & 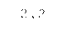 & $\because, ?$ & 2,5 & $: 9$ & $4,:$ & 1.8 & 1.9 & 1,8 & 2,1 & 2.1 & 2,3 & & 2.2 & 2,1 & 0.9 & 1.0 \\
\hline 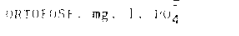 & 0.15 & 0.10 & $0.2 \%$ & * & 0 & $i n, 1$ & 0.1 & 11.05 & 0.18 & 0,23 & 0,12 & 0.05 & $0,0: 1$ & 0.04 & 0.04 & 0.05 \\
\hline 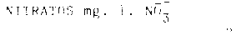 & 1,3 & 1,1 & 1,3 & 1,1 & $\therefore 1$ & 1,5 & 1.5 & 2 & $=$ & 1.5 & 2.4 & 2.6 & 2.8 & 3 & 0 & * \\
\hline Natratus mig. J. N1:? $\times 10^{-2}$ & 4,2 & 1,2 & 4,2 & 3,9 & 3,9 & $\therefore, 6$ & 2,6 & 2.6 & 2.6 & 2.6 & 2.6 & 7.2 & 7,2 & 3.2 & $x$ & 2.2 \\
\hline CTOHI.Fes m.g. $1.61^{-}$ & 6,7 & 11,3 & 12.7 & 12,7 & 12,7 & 11,3 & 11,3 & 12,7 & 12,7 & 11.3 & 12,7 & 11.3 & 11.3 & 13,3 & 7,1 & 8,5 \\
\hline GD.FATOS mg. I. $\mathrm{SO}_{4}^{-}$ & 6 & 6 & 6.5 & 0 & si & 5 & s & 5 & 5 & 4.9 & 5,2 & 6 & 6 & i & 2 & 5,8 \\
\hline $\mathrm{m} \times \mathrm{KF} 7 \mathrm{mp} \ldots 1 \cdot \mathrm{Co}_{3} \mathrm{ca}$ & 113 & $1: 2$ & 110 & 115 & 120 & 131 & 125 & 120 & 125 & 122 & $17 x$ & 119 & 123 & 123 & 92 & 105 \\
\hline 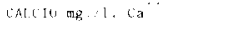 & 42,4 & 37,2 & 39,6 & 38,8 & 90 & $45, n$ & 43,2 & 42,4 & 42,4 & 42.4 & 44 & 42,8 & 46 & 46 & 32,5 & 36.8 \\
\hline$T \cdot A \cdot C \cdot m \cdot 1 \cdot m_{3} \mathrm{Ca}$ & $: 106$ & 94 & 108 & 100 & 104 & 106 & 110 & $1: 0$ & 112 & 112 & 112 & 110 & 110 & 112 & 90 & 98 \\
\hline 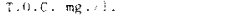 & 2,9 & $2, \mathrm{B3}$ & 2,88 & 2,89 & 3,08 & 3,16 & $\therefore 8$ & 2,72 & 2,72 & 2.72 & 2.68 & & & & & \\
\hline
\end{tabular}

calidad del agua (Miranda Braga, A. 1985; Alonso Fernández, J.R. 1985).

En la figura 3 podemos comprobar la evolución del caudal del río Piloña a lo largo de 1984 (meses de enero a septiembre), datos facilitados por la Comisaría de Aguas del Norte de España en la estación de aforos n. 303 de Villamayor, que se corresponde con nuestra E-33 de muestreo. Podemos observar el signi- ficativo y lógico descenso del mismo en el verano y la poca variación del régimen hidrológico en invierno y primavera.

\section{DISCUSION}

Para establecer los efectos producidos por los verti- 

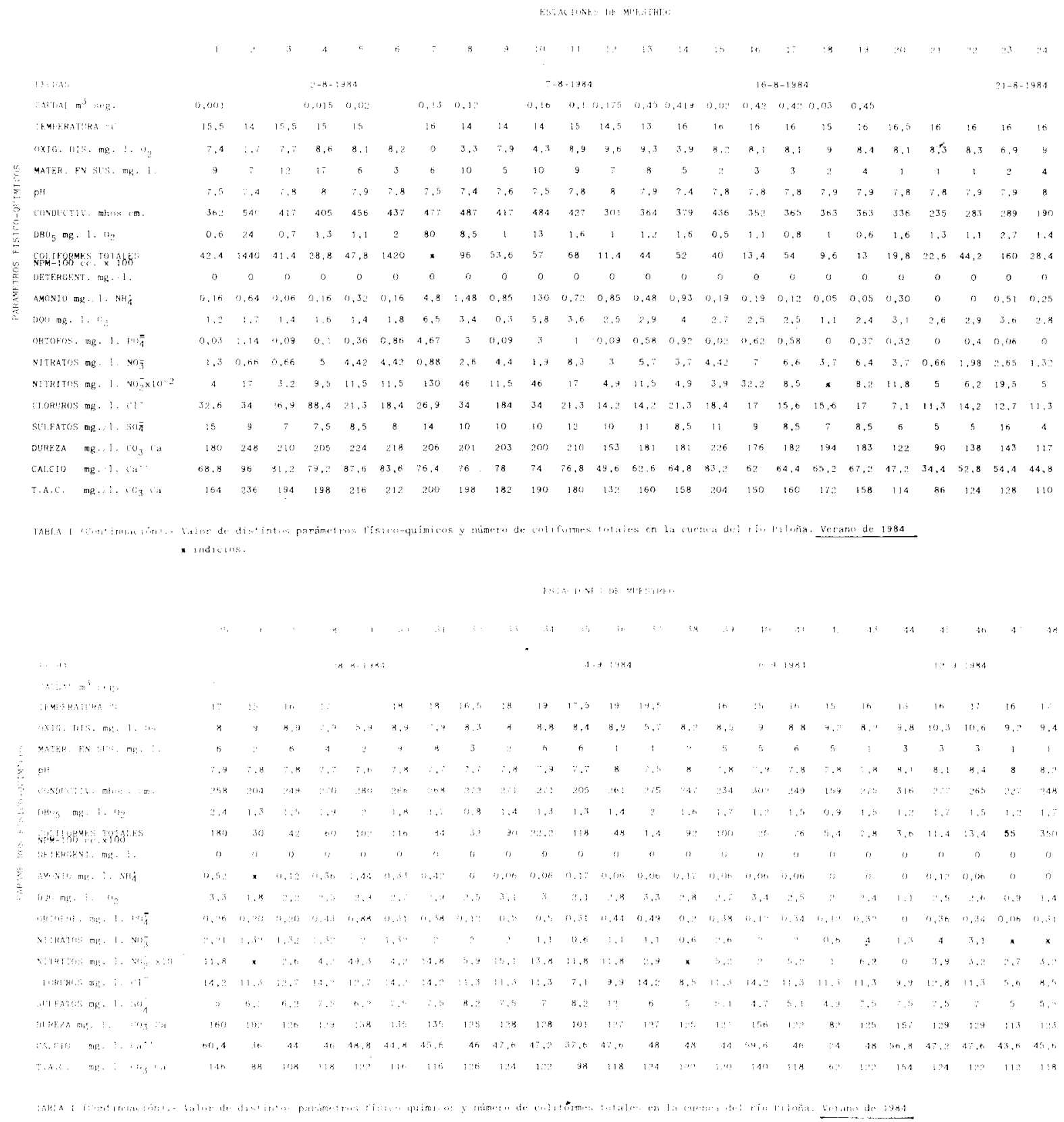

dos contaminados debemos remitirnos de nuevo a las tablas 1 y 2 y figura 4 y relacionar el deterioro de la composición química del agua con la alteración producida en la comunidad.

El número de coliformes totales se ha incrementado en las localidades que reciben vertidos importantes y que corresponden en su totalidad a estaciones situadas aguas abajo de núcleos urbanos.

En la estación 2 (arroyo prau Modesta), el número de coliformes totales es muy elevado; a la vez se registran valores de $\mathrm{O}$, disuelto inferiores a $2 \mathrm{mg} . / 1$, y un contenido en $\mathrm{DBO}_{\xi}$ elevado, condiciones que inducen una proporción de ciliados, cianofíceas, euglenoficeas, así como a una ausencia permanente de invertebrados de los órdenes plecóptera, efemeróptera,tricóptera, dando lugar a un bentos pobre, limitado a organismos poco exigentes de elevadas concentraciones de oxígeno. 


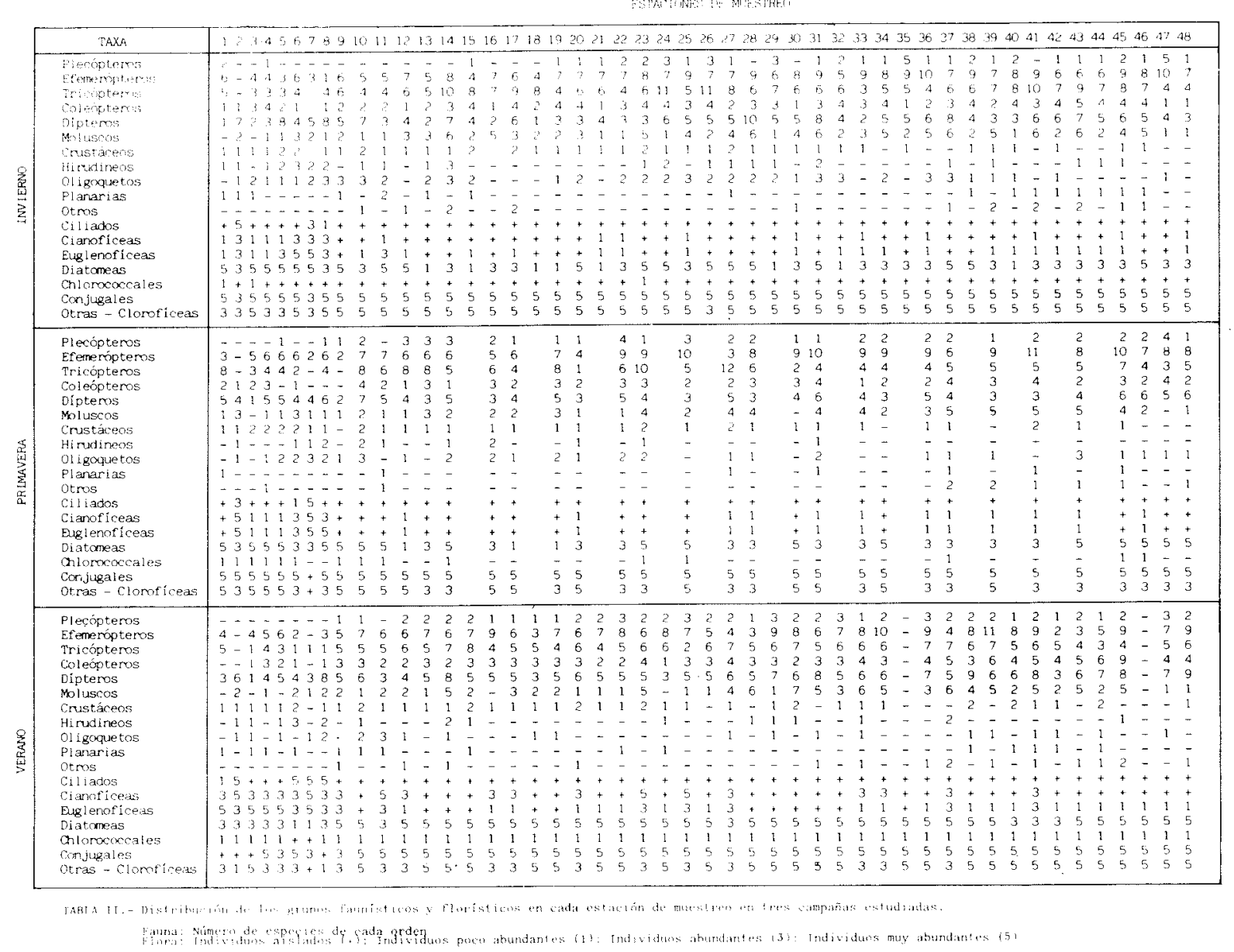

En la estación 7 se produce una situación semejante, pero aún mas exagerada, los vertidos de Nava arrojan al río una elevada carga contaminante que facilita la vida de un número incontable de coliformes, la ausencia de $O$, y la elevada concentración de $\mathrm{NH}^{+}, \mathrm{DBO}_{5}$ y DŌOO sólo permite la presencia de unos pocos quironómidos del grupo thumni y algunos tubifícidos; los ciliados, cianofíceas y euglenofíceas se encuentran en elevado número en estas condiciones que favorecen su desarrollo.

Poco antes de su confluencia con el río Fuensanta, el río Viao experimenta una importante recuperación de la calidad de sus aguas. descendiendo la concentración de $\mathrm{DBO}_{5}$ y $\mathrm{NH}^{+}$, recuperación que también afecta a las comunidades floristicas y faunisticas allí presentes. El río Fuensanta con las aguas de buena calidad en las tres campañas de muestreo, junto con el río Viao forman el río Pilona, que a su vez manticne elevados indices de calidad desde su nacimiento (figura 4).

En la E-23 localizada a la salida de Infiesto, se de- tecta asimismo un incremento en el número de coliformes totales, así como un ligero descenso en el contenido de $\mathrm{O}$, disuelto, debido a los vertidos de esta localidad; sin embargo la propia dinámica del río consigue que el impacto producido esté localizado en las inmediaciones de los diversos colectores, desapareciendo el efecto pocos metros más abajo donde ya se asienta una comunidad bastante compleja, con representantes de los órdenes Plecóptera, Efemeróptera y de la familia Heptageniidae, tricóptera y un abundante número de diatomeas y conjugales.

En la E-30 los vertidos de Villamayor producen un incremento de coliformes totales, mientras que los parámetros químicos y la flora y fauna no sufren alteraciones significativas.

La E-30 ya localizada en el rio Sella después de que el Piloña vierta sus aguas en él, presenta un elevado número de coliformes totales, sin que los parámetros químicos se vean alterados. ni tampoco la flora y la fauna respecto a estaciones anteriores, a pesar 
de haber recibido directamente los vertidos procedentes de la villa de Arriondas.

La presencia de maerófitos es significativamente menor en las primeras estaciones que en el cauce medio del río, y la proporción de estos aumenta de una manera clara cerca de la desembocadura.

En las márgenes de todos los ríos que constituyen la cuenca del Piloña se puede hablar de que la aliseda o bosque ribereño cubre prácticamente toda la orilla, salvo determinadas excepciones en que se ha deforestado para efectuar obras. En las estaciones 2, 6 y 7 es muy importante la presencia de especies ruderales; la estrecha relación observada en este caso entre el estado del agua, y la degradación estética de sus márgenes mediante escombros, desperdicios, etc., encuentra explicación en que a lo largo de toda la cuenca del río Piloña, todos son arrojados directamente al agua.

\section{CONCLUSIONES}

En líneas generales, a la vista de las tablas 1 y 2 y de la figura 4 , podemos deducir que la contaminación de las aguas del río Viao, es relativamente pequeña hasta Nava. A la salida de esta localidad, los numeroos vertidos que recibe el río afectan negativamente las condiciones físico-químicas bacteriólogicas, la flora y la fauna de sus aguas, circunstancia que se agrava sensiblemente en verano.

Debe de resaltarse el hecho de que a lo largo de todo su recorrido la calidad del agua, reflejada por los índices utilizados, se mantiene dentro de los límites admitidos por los distintos autores para definir aguas de buena calidad (Mingo, J., 1980; Verneaux, J. \& Tuffery, G., 1967; Dresscher, G.N. \& Mark, H., 1976), etc. Ni los vertidos de aguas residuales de numerosos colectores urbanos e industriales de Infiesto, ni los de Villamayor, Sevares y Arriondas, que como se decía en la introducción eran los núcleos de población más importantes, consiguen que la calidad del agua del río Piloña descienda hasta niveles que se pudieran considerar alarmantes. El río, por otra parte, se recupera muy rápidamente gracias a las continuas aportaciones de aguas limpias procedentes de todos los afluentes, lo que ocasiona que la desembocadura del río Piloña en el Sella no produzca en éste alteraciones en su nivel de calidad y las que efectivamente existen, una vez unidos los dos ríos, son debidas ex- clusivamente a los vertidos que Arriondas emite directamente a ambos.

\section{BIBLIOGRAFIA}

Alonso Alvarez, J.R. 1985. Utilización de algas y algunos macrófitos como indicadores biológicos de la calidad del agua en el río Viao-Piloña (Asturias). Com. en el III Congreso Español de Limnología (León).

Armitage, P.D. et al., 1983. The performance of a new biological water quality score system based on macroinvertebrates over a wide range of impolluted running water sites. Water Res. 17(3): 333-347.

Cairns, J. et al., 1968. The secuential comparison index -a simplified method for non biologist- to stimate relative differences in biological diversity in stream pollution studies. Jowrn. Wat. Poll. Contr. Feder 40(9): $1.607-1.613$.

Chandler, J.R. 1970. A biological approach to water quality management. Wat. Pollut. Cont 69: 415-422. Degremont, 1979. Manual técnico del agua. Art. Gráf. Grijelmo S.A. Bilbao.

Dresscher, G.N. \& Mark, H. 1976. A simplified method for the biological assesment of the quality of fresh and slightl brackish water. Hydrobiologia. 48(3): 199-201.

González Alvarez-Buylla, F. et al., 1985. La calidad del agua en la cuenca del río Piloña (Asturias). Actas del 111 Congreso Español de Limnologia. (en prensa). Mingo, J. 1980. Indices de calidad del agua. Ministerio de Obras Públicas.

Miranda Braga, A. 1985. Utilización de los macroinvertebrados benticos en la determinación de la calidad de las aguas en el río Viao-Piloña. Com. en $I I I$ Congreso Español de Limnología. Léón.

Shannon, C.E. \& Weaver, W. 1963. The mathematical theory of communication. Univ. Illinois Press. Ur. bana.

Standard Methods for the examination of water and wastewater. 1980. APHAAWWA-WPCF. Amer. Pub. Health Ass. Washington.

Verneaux, J. \& Tuffery, G. 1967. Une méthode zoologique pratique de détermination de la qualité biologique des eaux courantes. Indices biotiques. Annals. scient. Univ. Besançon. Zoologie. 3: 79-90. 